\title{
The Nonlinear Class-E Amplifier: a Case Study of Harmonic Balance Applied to Switched Circuits
}

\author{
Jordi Bonet-Dalmau, Pere Palà-Schönwälder, Francisco del-Águila-López and F. Xavier Moncunill-Geniz \\ Department of Signal Theory and Communications \\ Technical University of Catalonia (UPC) \\ Manresa 08242, Catalonia, Spain \\ jordi.bonet@upc.edu
}

\begin{abstract}
Nonlinear switched circuits are usually analyzed in the time domain. The use of frequency-domain methods to analyze the steady-state of switched circuits is usually restricted to linear circuits and, although Volterra series approaches are applied to the distortion of nonlinear switched circuits, the use of harmonic balance (HB) methods in nonlinear switched circuits seems to be marginal if not discarded. In this paper, we analyze the nonlinear class-E amplifier with an ideal switch by means of the HB method. We show how the switching function may be carefully chosen to improve the accuracy of the solution. When the circuit achieves actual class-E operation, the method converges better and is more accurate than classical timedomain methods. We conclude that the $\mathrm{HB}$ method is an alternative to time-domain methods that can be used to analyze the class-E amplifier in its expected operation mode.
\end{abstract}

\section{INTRODUCTION}

Several approaches have been used to analyze nonlinear switched circuits (see [1] and [2] and references therein). The advantages of using ideal switches in circuit simulation are reported in [3]. Most of these methods work in the time domain. Only a few -most under certain restrictions such as the linearity of the circuit- have been developed in the frequency domain. Harmonic balance (HB) methods use a linear combination of sinusoids to describe the steady state solution in the frequency domain while, for convenience, nonlinearities are usually evaluated in the time domain [4][6]. Volterra series approaches avoid the cost of evaluation in the time domain [7]. However, these approaches require substantial ad-hoc handwritten work and are targeted at circuits with mild nonlinearities.

It has been assumed that the application of HB methods to periodically switched nonlinear circuits is computationally expensive, due to the large number of frequency components caused by both nonlinearities and switching [2]. However, class-E amplifiers operating in class-E mode show waveforms without jump discontinuities (see Fig. 7, top) which may be well approximated as a linear combination of sinusoids. On this basis, we used the HB method as described in [4] to analyze the class-E amplifier, considering the switching function that controls the switch as an input. An investigation of the meaning of the switching function in a time-domain and frequency-domain context led to different switching functions showing a compromise between ringing and aliasing.

This work was supported by the Spanish Dirección General de Investigación under Grant TEC2006-12687/TCM.
The rest of this paper is organized as follows. Section II describes HB implementation for handling both nonlinearities and switching functions. Section III focuses on the different switching functions that are considered in this paper for use in combination with the HB method. Simulation results are shown in Section IV, while the conclusions are presented in Section V.

\section{THE HARMONIC BALANCE IMPLEMENTATION}

To analyze the class-E amplifier depicted in Fig. 1 we have used the piecewise $\mathrm{HB}$ method described in [4]. Although there is no mention in this reference -or, to our knowledge, in recent literature- about how to handle switches, we have considered the switching function $s(t)$ that controls the state of the switch as one more input, in addition to the DC voltage $v_{\mathrm{DD}}$.

In the HB approach, we can approximate the control variable of the nonlinearity $v(t)$ (of frequency $\omega_{0}$ and period $T_{0}$ ) by its truncated Fourier series as

$$
v(t)=\sum_{n=-\frac{N-1}{2}}^{n=\frac{N-1}{2}} V_{n} e^{j n \omega_{0} t},
$$

where $N$ is the total number of Fourier coefficients (FC). We define the frequency-domain vector of FC

$$
\mathbf{V}=\left[\begin{array}{lllllll}
V_{0} & V_{1} & \cdots & V_{\frac{N-1}{2}} & V_{-\frac{N-1}{2}} & \cdots & V_{-1}
\end{array}\right]^{\mathrm{T}},
$$

with $\mathrm{T}$ denoting the transpose vector. We also define the timedomain vector of $N$ equally spaced samples during one period

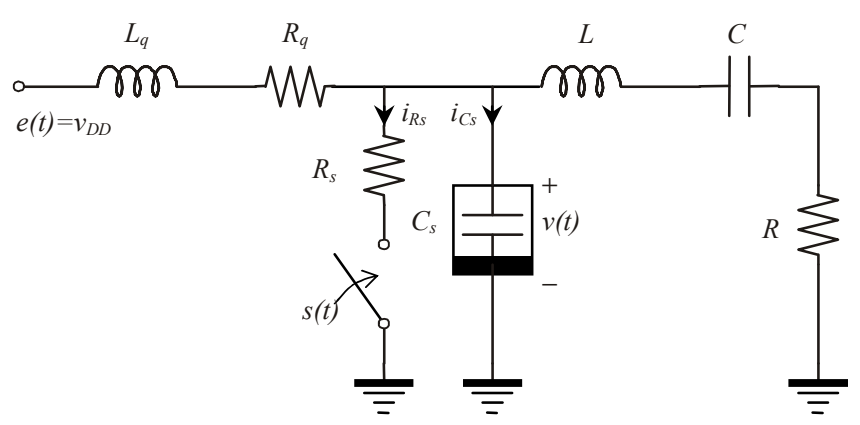

Figure 1. Class-E amplifier. The switching device is modeled by $R_{s}$, the nonlinear capacitance $C_{s}$ and the ideal switch, with $s(t)$ defined in (14). 


$$
\mathbf{v}=\left[\begin{array}{llll}
v_{0} & v_{1} & \cdots & v_{N-1}
\end{array}\right]^{\mathrm{T}}, \text { with } v_{m}=v\left(t_{0}+m \frac{T_{0}}{N}\right),
$$

with $t_{0}$ being a time origin. We can compute (2) from (3) via the Discrete Fourier Transform (DFT), or (3) from (2) via the Inverse Discrete Fourier Transform (IDFT).

The HB formulation of the circuit in Fig. 1 yields the following system of $N$ equations

$$
V_{n}+Z_{n}\left(I_{C s n}(\mathbf{V})+I_{R s n}(\mathbf{V}, \mathbf{s})\right)=E H_{n}, n=-\frac{N-1}{2}, \cdots, \frac{N-1}{2},
$$

where the impedances $Z_{n}$ are given by

$$
Z_{n}=\frac{R_{q}-n^{2} \omega_{0}^{2}\left(R_{q} L+R L_{q}\right) C+j n \omega_{0}\left(R_{q} R C+L_{q}\left(1-n^{2} \omega_{0}^{2} L C\right)\right)}{1-n^{2} \omega_{0}^{2}\left(L+L_{q}\right) C+j n \omega_{0}\left(R+R_{q}\right) C}
$$

and, in accordance with the notation in (3), $\mathbf{s}$ is the vector of samples of the switching function $s(t), E H_{n}$ is the product of the FC of the constant input $e(t)$ and a network function (which is zero except for $E H_{0}=v_{\mathrm{DD}}$ ), $I_{C s n}$ are the FC of the current flowing through $C_{s}$ defined by [8]

$$
i_{C s}(t)=\frac{\partial q(t)}{\partial t}=\frac{\partial}{\partial t}\left(\frac{1}{1-\gamma} C_{j 0} V_{b i}\left(1+\frac{\nu(t)}{V_{b i}}\right)^{1-\gamma}\right),
$$

or, alternatively [8],

$$
i_{C s}(t)=\frac{\partial q(t)}{\partial v} \frac{\partial v(t)}{\partial t}=C_{j 0}\left(1+\frac{v(t)}{V_{b i}}\right)^{-\gamma} \frac{\partial v(t)}{\partial t},
$$

and $I_{R s n}$ are the $\mathrm{FC}$ of the current flowing through $R_{s}$

$$
i_{R s}(t)=\frac{v(t)}{R_{s}} s(t) .
$$

When defining $\mathbf{Z}$ as a diagonal matrix with its diagonal elements consisting of $Z_{n}$ ordered in coherence with (4), we can write (4) in a compact form as the system of equations

$$
\mathbf{V}+\mathbf{Z}\left(\mathbf{I}_{C s}(\mathbf{V})+\mathbf{I}_{R s}(\mathbf{V}, \mathbf{s})\right)=\mathbf{E},
$$

which must be solved for $N$ unknowns in vector $\mathbf{V}$. Although this is not the general case, the nonlinear part of the circuit in (9), $\mathbf{I}_{C s}$, is separated from the part in which the ideal switch participates, $\mathbf{I}_{R s}$. Thus, different techniques can be used to compute each part. However, from a computational point of view, it is more efficient to treat both parts as one, by defining

and rewriting (9) as

$$
i(t)=i_{C S}(t)+i_{R s}(t),
$$

$$
\mathbf{V}+\mathbf{Z} \mathbf{I}(\mathbf{V}, \mathbf{s})=\mathbf{E} \text {. }
$$

As the notation in (11) indicates, $\mathbf{I}$ must be computed from $\mathbf{V}$ and $\mathbf{s}$. This is done as follows. We compute the vector $\mathbf{s}$ once at the beginning of the iterative resolution process. Then, at each iteration, we compute $\mathbf{v}$ from $\mathbf{V}$ via IDFT. Next, we compute $\mathbf{i}$ in the time-domain from $\mathbf{v}$ and $\mathbf{s}$, according to equations (6) -or (7) - and (8) and, finally, we compute I from i via DFT. It follows that each evaluation of (11) implies one IDFT followed by one DFT.

In the HB method, aliasing avoidance techniques are normally used when evaluating nonlinearities (6) or (7) in the time domain. Such techniques are required mainly for dealing with hard nonlinearities. The same technique is useful for improving the evaluation of (8) in the time domain. It consists in artificially increasing the total number of FC, by adding $k N$ (with $k$ usually integer) zero coefficients,

$$
\mathbf{V}^{k}=\left[\begin{array}{llllllllll}
V_{0} & V_{1} & \cdots & V_{\frac{N-1}{2}} & 0_{1} & \cdots & 0_{k N} & V_{-\frac{N-1}{2}} & \cdots & V_{-1}
\end{array}\right],
$$

so the total number of FC is $(1+k) N$. The IDFT of $\mathbf{V}^{k}$ produces $(1+k) N$ samples:

$$
\mathbf{v}^{k}=\left[\begin{array}{llll}
v_{0}^{k} & v_{1}^{k} & \cdots & v_{(1+k) N-1}^{k}
\end{array}\right] .
$$

In our approach, $\mathbf{s}^{k}$ also has $(1+k) N$ samples. However, these are not computed by artificially inserting a number of zero FC, but by directly sampling $s(t)$ or using the truncated Fourier series of $s(t)$, as will be seen in Section III. From $\mathbf{v}^{k}$ and $\mathbf{s}^{k}$, we computed $\mathbf{i}^{k}$ and via DFT its $(1+k) N$ FC, which are truncated to $N$ to finally obtain $\mathbf{I}$. These FC have less aliasing than those obtained when only $N$ samples are used in the time domain.

\section{SAMPLING The SwITCHING FUnCTION}

In the following sections, we will consider the switching function $s(t)$ with a duty cycle of $40 \%, D=0.4$, defined as

$$
s(t)=\left\{\begin{array}{cc}
1 & p T_{0}<t \leq D T_{0}+p T_{0} \\
0 & D T_{0}+p T_{0}<t \leq T_{0}+p T_{0}
\end{array}, \quad p \text { integer },\right.
$$

or, from the FC, $S_{n}$, of its Fourier series, as

$$
s(t)=\sum_{n=-\infty}^{n=\infty} S_{n} e^{j n \omega_{0} t}, \text { with } S_{n}=e^{-j n \pi D} \frac{\sin (n \pi D)}{n \pi} .
$$

\section{A. $\quad$ Truncated Fourier series approach}

In the HB method, the idea is to compute $N$ FC of the truncated Fourier series of the solution (1) with maximum accuracy. In accordance with this, all inputs of the circuit, including the switching function, should be defined with $N$ FC of its truncated Fourier series. Following this criterion, we propose using this first switching function:

$$
S_{1}(t)=\sum_{n=-\frac{N-1}{2}}^{n=\frac{N-1}{2}} S_{n} e^{j n \omega_{0} t}
$$

Figure 2 shows some ringing in $s_{1}(t)$ as a result of the truncation for $N=15$. Sampling $s_{1}(t)$ as in (3), for different values of $t_{0}$, results in the following time-domain vectors of samples: $\mathbf{s}_{1, \mathrm{o}}$ taking $t_{0}=T / 2 N$ (circle samples in Fig. 2) and $\mathbf{s}_{1, \Delta}$ taking $t_{0}=T / N$ (triangle samples in Fig. 2), which may have exhibited less $\left(\mathbf{s}_{1,0}\right)$ or more ringing $\left(\mathbf{s}_{1, \Delta}\right)$. In this approach, there is no aliasing in the $\mathrm{FC}$ and no restriction in the relation between $D, N$ and $t_{0}$, in contrast with the following approach.

\section{B. Time-domain sampling approach}

Although the HB is a frequency-domain method, we have seen in the previous section that the switching function is used in the time domain. Now, it is worth considering how a pure time-domain method would work. Samples of $s(t)$, which are all 1 during the ON phase and all 0 during the OFF phase, would be taken. These samples can be used to build the vector $\mathbf{s}_{2, \mathrm{O}}$ (free of ringing), taking $t_{0}=T / 2 N$ (circle samples in Fig. 3). 
We denote as $S_{n}{ }_{n}$ the FC computed via DFT from $\mathbf{s}_{2,0}$. Note that, in this case, these FC have aliasing. Next, we define $s_{2}(t)$ as in (16), substituting $S_{n}$ with $S_{n}{ }_{n}$. Sampling $s_{2}(t)$, taking different values of $t_{0}$, as $\mathbf{s}_{2, \Delta}$ with $t_{0}=T / N$ (triangle samples in Fig. 3), is of no practical interest as ringing is present.

The vector of samples $\mathbf{s}_{2, \mathrm{o}}$ in Fig. 3 has been obtained with such a combination of $D, N$ and $t_{0}$ that discontinuities lie exactly between two samples. This is a restriction. Changing $t_{0}$ to a quantity does not change the vector of samples (although a rotation may occur). So, it creates a phase shift of the same quantity between $s(t)$ and $s_{2}(t)$ and in the computed solution (1) as well. In addition, if $N$ is not well-chosen, the number of ones and zeros in the vector of samples may change, with worse consequences than those of a phase shift.

To avoid the uncertainty in the situation of the jump discontinuity when sampling $s(t)$, we can use a combination of $D, N$ and $t_{0}$, so that samples coincide with the discontinuities. We can then fix the value of these samples to the value that $s_{1}(t)$ takes at the discontinuities (which is approximately 0.5 ), see $\mathbf{s}_{3,0}$ (taking $\left.t_{0}=T / N\right)$ in Fig. 4 . Note that $s_{3}(t)$ shows less ringing than $s_{1}(t)$ and $s_{2}(t)$, while the slope of the discontinuities is smaller (see Fig. 4).

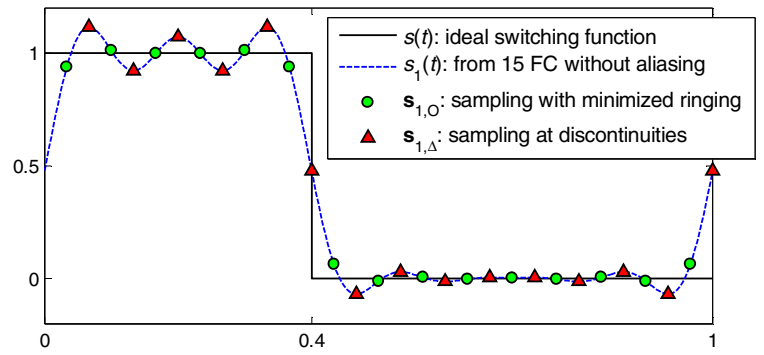

Figure 2. Switching function $s_{1}(t)$ as defined in (16).

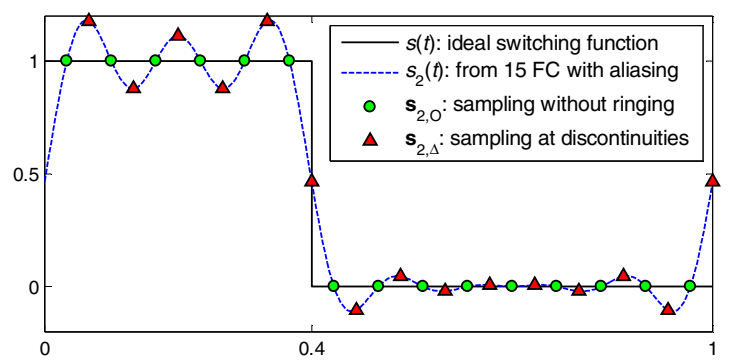

Figure 3. Switching function $s_{2}(t)$ computed from the FC obained via DFT from the $N=15$ samples of the vector $\mathbf{s}_{2,0}$.

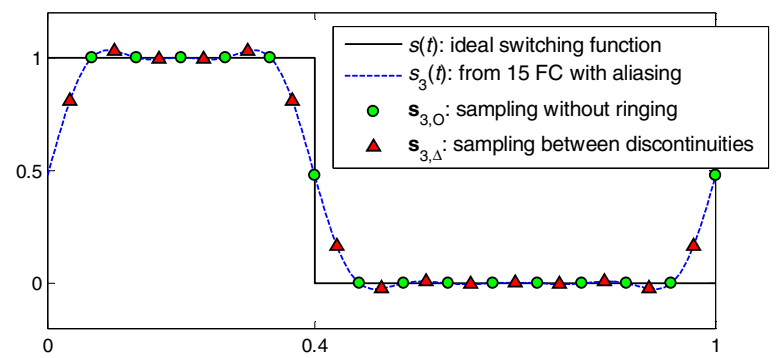

Figure 4. Switching function $s_{3}(t)$ computed from the FC obained via DFT from the $N=15$ samples of the vector $\mathbf{s}_{3,0}$.

\section{Discussion}

To assess the performance of the above vectors of samples, we may first consider the case in which the switching function is the input signal of a comparator with a threshold level a little lower than unity. This is an extreme case of hard nonlinearity. As the samples at the comparator output can only take two values, $\mathrm{V}_{\mathrm{ON}}$ and $\mathrm{V}_{\mathrm{OFF}}$, it is clear that they will be well computed if they result in a $\mathrm{V}_{\mathrm{ON}}$ value in the ON phase and a $\mathrm{V}_{\mathrm{OFF}}$ value otherwise. With a threshold level of $0.93, \mathbf{s}_{1, \Delta}, \mathbf{s}_{2, \Delta}$ and $\mathbf{s}_{3, \Delta}$ produce some incorrect samples at the output. In contrast, $\mathbf{s}_{1, \mathrm{O}}$ produces correct output samples for this threshold level (but as the threshold level approaches unity, some output samples are incorrect). The vector $\mathbf{s}_{2,0}$ always produces correct output samples for any threshold level. Finally, using the vector $\mathbf{s}_{3,0}$ leads to a single incorrect sample (at one discontinuity) for all threshold levels.

Next, we consider a linear circuit, which may be seen as the limiting case of soft nonlinearity. In this case, samples of $s_{1}(t)$ allow the exact computation of the $N$ FC of the solution, provided that aliasing is avoided by taking $k=1$. In contrast, samples of $s_{2}(t)$ and $s_{3}(t)$ will never allow exact computation although aliasing can be reduced by using higher $k$ resulting in more accurate solutions. In this case, it makes no sense to differentiate between $\mathbf{s}_{\mathrm{q}, \mathrm{O}}$ and $\mathbf{s}_{\mathrm{q}, \Delta},(q=[1,2,3])$ as they come from the same FC. As the vectors $\mathbf{s}_{\mathrm{q}, \Delta}$ perform as well as the vectors $\mathbf{s}_{\mathrm{q}, \mathrm{o}}$, from the aliasing criterion point of view, while performing worse from the ringing criterion point of view, it follows that the vectors $\mathbf{s}_{\mathrm{q}, \Delta}$ may be discarded.

To conclude, in a linear switched circuit, the aliasing criterion is the only one worth applying and samples of $s_{1}(t)$ should be used, while in a nonlinear switched circuit a compromise between aliasing and ringing exists, as the kind of nonlinearity decides the outcome. As a rule of thumb, in a soft nonlinearity, $\mathbf{s}_{1,0}$ is expected to produce better results, while in a hard nonlinearity, like a comparator, $\mathbf{s}_{2, \mathrm{o}}$ or $\mathbf{s}_{3, \mathrm{o}}$ may perform better.

\section{Simulation ResUlts}

We have analyzed the circuit in Fig. 1 using the described technique for the parameters in [8]: $V_{D D}=42 \mathrm{~V}, L_{q}=97 \mu \mathrm{H}$, $R_{q}=10 \Omega, \quad R=24 \Omega, L=6.2 \mu \mathrm{H}, \quad C=138 \mathrm{pF}$. The nonlinear capacitor is defined by $R_{s}=2.4 \Omega, C_{j 0}=230 \mathrm{nF}, V_{b i}=0.7127 \mathrm{~V}$ and $\gamma=2 / 3$. The switching function has a frequency $f_{0}=6.78 \mathrm{MHz}$ and a duty cycle $D=0.4$. Values have been normalized to obtain a period of one second.

The system of equations (11) has been solved using fsolve from MATLAB, with an initialization carried out with the linear solution (i.e., $\gamma=0$ ) of the circuit. Convergence is achieved in a few iterations, without providing the Jacobian to fsolve or having to use any continuation technique. We have evaluated the accuracy of the method by computing the root mean square error (RMSE) of the solutions against a reference computed using this method with $N \sim 1000$ (which is indistinguishable from the PSpice solution computed with about 1000 samples).

When no aliasing avoidance is used (i.e., $k=0$ ), vectors $\mathbf{s}_{\mathrm{q}, \mathrm{O}}$ result in an appreciably lower RMSE than vectors $\mathbf{s}_{\mathrm{q}, \Delta}$ (for a 
fixed $q$ ), while the use of (7) instead of (6) to evaluate the current flowing through $C_{s}$ results in a slight reduction in the RMSE. When $k>0$, making use of (6) or (7) does not change the accuracy of the solution. Hence, in the following description, we will consider the use of $\mathbf{s}_{\mathrm{q}, \mathrm{o}}$ and (7).

In this particular circuit, we can separately apply antialiasing to (7) and (8) to test its effect individually. We verify that the aliasing introduced by (7) becomes irrelevant, taking $k=1$. This is due to the fact that the linearity is relatively soft. The effect that $k$ has on (8) depends on the switching function under consideration. If we consider $\mathbf{s}_{1,0}$, the use of $k=1$ is enough to wipe out the effects of aliasing. Viewing (8) in the frequency domain as the convolution of the $N \mathrm{FC}$ of $v(t)$ and the $2 N$ free-of-aliasing FC of $s_{1}(t)$, the $N$ FC obtained from the truncation of this convolution have no aliasing at all, either from the switching function or from the product in (8). Obviously, when $\mathbf{s}_{1, \mathrm{o}}$ is used, no improvement is obtained with $k>1$, while when $\mathbf{s}_{2,0}$ or $\mathbf{s}_{3,0}$ are used, higher values of $k$ yield lower RMSE (see Fig. 5). Moreover, the use of any switching function with no aliasing avoidance, i.e. $k=0$, is not an option when compared with $k=1$, considering the small reduction in the computing time $(30 \%, N=75)$ at the expense of a dramatic increase in the RMSE (70\% with $\mathbf{s}_{1,0}$ and $300 \%$ with $\mathbf{s}_{2,0}$ and $\left.\mathbf{s}_{3,0}, N=75\right)$.

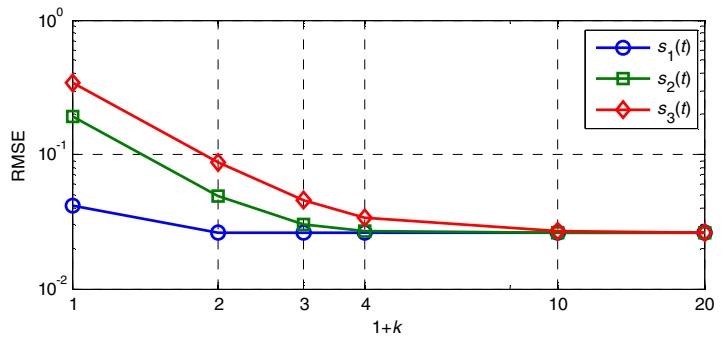

Figure 5. RMSE as a function of the aliasing avoidance performed with $(1+k) N$ samples in the time domain. $k=0$ means no aliasing avoidance. $N=75$.

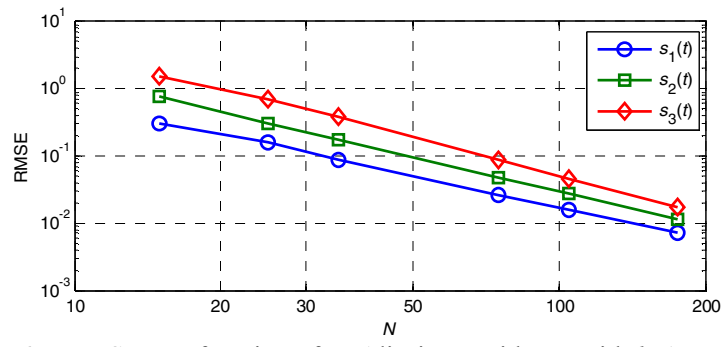

Figure 6. RMSE as a function of $N$. Aliasing avoidance with $k=1$.
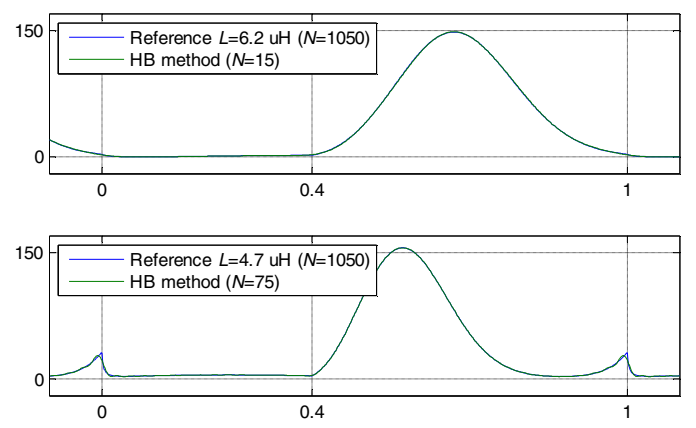

Figure 7. Waveform of $v(t) . L=6.2 \mu \mathrm{H}$ (top) and $L=4.7 \mu \mathrm{H}$ (bottom).
Figure 6 shows that the RMSE of the computed solutions with $k=1$ is inversely proportional to approximately $N^{1.6}$ for all switching functions. The vector $\mathbf{s}_{1,0}$ gives the most accurate solutions, while $\mathbf{s}_{3,0}$ gives the least accurate ones. As a rule of thumb, $\mathbf{s}_{2, \mathrm{O}}$ needs $50 \%$ more samples than $\mathbf{s}_{1, \mathrm{O}}$ to achieve the same RMSE.

With the same number of samples, the HB method described here (considering $\mathbf{s}_{1,0}$ with $k=1$ ) is much more accurate and converges more quickly than the time domain method described in [8]. In turn, this time-domain method is much more accurate than PSpice, with a comparable number of samples. As an example, the computed relative mean square error (MSE) using this HB method is $1.61 \cdot 10^{-7}$ after 17 iterations (with $N=75$ ), while it is $2.96 \cdot 10^{-4}$ after 32 iterations, providing the Jacobian in [8] (with 100 equations and 82 samples) and $7.59 \cdot 10^{-3}$ in PSpice (with 100 samples). These results must be balanced with those obtained when jump discontinuities appear in the solution, as when $L=4.7 \mu \mathrm{H}$ (see Fig. 7, bottom). In this situation, the relative MSE using this $\mathrm{HB}$ method has increased to $9.68 \cdot 10^{-5}$, while [8] yields a relative MSE of $2.29 \cdot 10^{-4}$ (i.e., essentially unchanged).

\section{CONCLUSIONS}

In this paper, we have analyzed the nonlinear class-E amplifier using the HB method. We gain some insight into the conceptual differences between time-domain and frequencydomain methods, to justify the selection of the switching function that has its FC free of aliasing. Simulation shows how other switching functions require at least 50\% more samples to achieve the same RMSE. Simulation results show advantages in terms of convergence and accuracy of the HB method in comparison with time-domain methods when the circuit operates in class-E mode.

\section{REFERENCES}

[1] J. Vlach and A. Opal, "Modern CAD methods for analysis of switched networks," IEEE Trans. Circuits Syst. I, Fundam. Theory Appl., vol. 44, no. 8, pp. 759-762, Aug. 1997.

[2] F. Yuan and A. Opal, "Computer methods for switched circuits," IEEE Trans. Circuits Syst. I, Fundam. Theory Appl., vol. 50, no. 8, pp. 10131024, Aug. 2003.

[3] A. Opal and J. Vlach, "Consistent initial conditions of nonlinear networks with switches," IEEE Trans. Circuits Syst., vol. 38, no. 7, pp. 698-710, July 1991.

[4] C. Camacho-Peñalosa, "Numerical steady-state analysis of nonlinear microwave circuits with periodic excitation," IEEE Trans. Microw. Theory Tech., vol. 31, no. 9, pp. 724-730, Sep. 1983.

[5] K. Kundert, J. K. White, and A. Sangiovanni-Vincentelli, Steady-State Methods for Simulating Analog and Microwave Circuits. Norwell, MA: Kluwer, 1990.

[6] A. A. Buonomo and A. Lo Schiavo, "A constructive method for finding the periodic response of nonlinear circuits," IEEE Trans. Circuits Syst. I, Fundam. Theory Appl., vol. 50, no. 7, pp. 885-893, July 2003.

[7] F. Yuan and A. Opal, "Distortion Analysis of periodically switched nonlinear circuits using time-varying Volterra series," IEEE Trans. Circuits Syst. I, Fundam. Theory Appl., vol. 48, no. 6, pp. 726-738, June 2001.

[8] F. del-Águila-López, P. Palà-Schönwälder, P. Molina-Gaudó and A. Mediano-Heredia, "A discrete-time technique for the steady-state analysis of nonlinear class-E amplifiers," IEEE Trans. Circuits Syst. I, Reg. Papers, vol. 54, no. 6, pp. 1358-1366, June 2006. 